\title{
Distância interincisiva máxima em crianças respiradoras bucais
}

Débora Martins Cattoni*, Fernanda Dreux Miranda Fernandes**, Renata Cantisani Di Francesco***, Maria do Rosário Dias de Oliveira Latorre ${ }^{\star \star \star \star}$

\section{Resumo}

Introdução: a distância interincisiva máxima é um importante aspecto na avaliação miofuncional orofacial, pois distúrbios miofuncionais orofaciais podem limitar a abertura da boca. Objetivo: mensurar a distância interincisiva máxima de crianças respiradoras bucais, relacionando-a com a idade, e comparar as médias dessas medidas com as médias dessa distância em crianças sem queixas fonoaudiológicas. Métodos: participaram 99 crianças respiradoras bucais, de ambos os gêneros, com idades entre 7 anos e 11 anos e 11 meses, leucodermas, em dentadura mista. O grupo controle foi composto por 253 crianças, com idades entre 7 anos e 11 anos e 11 meses, leucodermas, em dentadura mista, sem queixas fonoaudiológicas. Resultados: os achados evidenciam que a média das distâncias interincisivas máximas das crianças respiradoras bucais foi, no total da amostra, de $43,55 \mathrm{~mm}$, não apresentando diferença estatisticamente significativa entre as médias, segundo a idade. Não houve diferença estatisticamente significativa entre as médias da distância interincisiva máxima dos respiradores bucais e as médias dessa medida das crianças do grupo controle. Conclusões: a distância interincisiva máxima é uma medida que não variou nos respiradores bucais, durante a dentadura mista, segundo a idade, e parece não estar alterada em portadores desse tipo de disfunção. Aponta-se, também, a importância do uso do paquímetro na avaliação objetiva da distância interincisiva máxima.

Palavras-chave: Face. Medidas. Boca. Criança. Respiração bucal.

\section{INTRODUÇÃO}

Um dos distúrbios miofuncionais orofaciais mais frequentes na clínica fonoaudiológica é a respiração bucal, destacada como tendo alta prevalência na população, em todas as idades ${ }^{10}$. A avaliação, bem como a detecção precoce, do indivíduo com respiração bucal é extremamente importante, pois tal distúrbio é considerado altamente comprometedor no desenvolvimento do processo nasomaxilar, devido à ruptura do equilíbrio fisiológico em que se baseia a arquitetura dentomaxilofacial. Os efeitos da respiração bucal têm sido muito discutidos na literatura, que aponta as alterações do desenvolvimento normal da face, ossos e oclusão, em função do desequilíbrio provocado nas relações entre os tecidos muscular, ósseo e

* Especialista em Motricidade Orofacial pelo Conselho Federal de Fonoaudiologia (CFFa). Mestre em Ciências pela Faculdade de Medicina da Universidade de São Paulo (FMUSP). Doutora em Ciências pela FMUSP.

** Professora associada do curso de Fonoaudiologia da FMUSP.

*** Doutora em Medicina pela Faculdade de Medicina da USP. Médica assistente doutora da divisão de Otorrinolaringologia do Hospital das Clínicas da FMUSP.

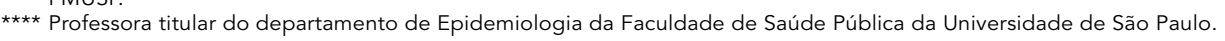


dentário. Dessa forma, evidenciam-se alterações no padrão esquelético e muscular dos indivíduos respiradores bucais i, $^{1,9,10,17,18,19,22,26,29}$.

Durante a avaliação fonoaudiológica, Bianchi$\mathrm{ni}^{3}$ propõe a utilização do paquímetro para mensurar a distância interincisiva máxima, isto é, a distância entre os incisivos superiores e inferiores, na maior abertura possível da boca. Tal instrumento também é utilizado para medir a abertura máxima da boca, considerada como a distância entre os incisivos superiores e os inferiores, somando-se a marcação da sobremordida ou subtraindo-se a distância da mordida aberta anterior.

Outros autores $20,21,23,24,27$ também preconizam a utilização do paquímetro para mensurar, entre outras medidas orofaciais, a abertura máxima da boca.

$\mathrm{O}$ uso do paquímetro, instrumento da antropometria, oferece inúmeras vantagens na avaliação objetiva do complexo craniofacial, por ser uma técnica simples, não-invasiva, sem risco para o paciente e com baixo custo ${ }^{30}$.

No que se refere aos índices de normalidade da abertura máxima de boca, Bianchini ${ }^{4}$ refere que a abertura inferior a $35 \mathrm{~mm}$ na criança é um alerta sobre possíveis problemas musculares e/ou articulares. Hamazaki et al. ${ }^{16}$ encontraram, em seu estudo com crianças de 6 a 12 anos de idade, que a média para a abertura da boca foi de $48,33 \mathrm{~mm}$, sendo que essa medida aumentou de acordo com a idade. Ríspoli e Bacha ${ }^{23}$ apontam que a abertura máxima de boca é de 40 a $45 \mathrm{~mm}$, não fazendo distinção entre os gêneros nem entre as idades. Rodrigues ${ }^{25}$ considera, em adultos, o valor de $40 \mathrm{~mm}$ como referência da amplitude da boca em abertura máxima.

Em um estudo, cujo objetivo foi descrever a distância interincisiva máxima em crianças leucodermas sem queixas fonoaudiológicas, em dentadura mista, foi verificado que a média dessa distância na amostra foi de $44,75 \mathrm{~mm}$, sendo observada diferença estatisticamente significativa somente no gênero masculino entre 7 e 11 anos e 8 e
11 anos de idade. As crianças do gênero feminino apresentaram médias inferiores às das crianças do gênero masculino, exceto aos 8 anos de idade ${ }^{6}$.

O interesse para a realização do presente estudo cresceu à medida que se observou a falta de referencial da distância interincisiva máxima em crianças respiradoras bucais, bem como da descrição desse aspecto de acordo com a idade.

Os objetivos desse estudo foram: (1) descrever a distância interincisiva máxima em crianças respiradoras bucais e verificar se há diferença estatisticamente significativa entre as médias dessa medida, segundo a idade; e (2) comparar as médias da distância interincisiva máxima dos respiradores bucais com as médias dessa medida de crianças sem queixas fonoaudiológicas e verificar se há diferença estatisticamente significativa entre as médias dessas duas populações, segundo a idade.

\section{MATERIAL E MÉTODOS \\ Casuística}

Foram avaliadas 99 crianças respiradoras bucais, na faixa etária entre 7 anos e 11 anos e 11 meses, sendo 51 do gênero masculino (51\%) e 49 do gênero feminino (49\%). A idade média foi de 8 anos e 5 meses e a mediana foi de 8 anos de idade. As crianças foram divididas de acordo com a idade: 31 crianças (31\%) de 7 anos a 7 anos e 11 meses; 21 crianças (22\%) de 8 anos a 8 anos e 11 meses; 21 crianças (21\%) de 9 anos a 9 anos e 11 meses; 12 crianças (12\%) de 10 anos a 10 anos e 11 meses; e 14 crianças (14\%) de 11 anos a 11 anos e 11 meses.

Os critérios de inclusão das crianças para esse estudo foram: (a) diagnóstico otorrinolaringológico de respiração bucal; (b) alteração funcional da respiração; (c) leucoderma; e (d) dentadura mista com a irrupção completa dos quatro primeiros molares permanentes.

Os critérios de exclusão das crianças para esse estudo foram: (a) histórico de tratamento fonoaudiológico prévio e/ou atual, independentemente da área (voz, linguagem, motricidade orofacial 
e/ou audição); (b) histórico de cirurgia facial e/ ou faríngea; (c) histórico de síndrome e/ou doença neurológica e/ou úvula bífida; (d) histórico de malformações craniofaciais; e (e) histórico de tratamento ortodôntico, ortopédico facial, craniomandibular e/ou de disfunção da articulação temporomandibular (ATM) prévios e/ou atuais.

O grupo controle foi composto por 253 crianças (mesma amostra do estudo de Cattoni ${ }^{5}$, 2003), na faixa etária entre 7 anos e 11 anos e 11 meses, sendo 137 do gênero masculino (54\%) e 117 do gênero feminino (46\%), leucodermas, em dentadura mista com a irrupção completa dos quatro primeiros molares permanentes, sem histórico de tratamento fonoaudiológico prévio e/ou atual, independentemente da área (voz, linguagem, motricidade orofacial e/ou audição), sem queixas fonoaudiológicas e frequentadoras de escolas particulares da cidade de São Paulo. A idade média foi de 8 anos e 5 meses e a mediana foi de 9 anos de idade.

As crianças foram divididas de acordo com a idade: 48 crianças $(18,9 \%)$ de 7 anos a 7 anos e 11 meses; 51 crianças $(20,5 \%)$ de 8 anos a 8 anos e 11 meses; 50 crianças $(19,7 \%)$ de 9 anos a 9 anos e 11 meses; 54 crianças $(21,3 \%)$ de 10 anos a 10 anos e 11 meses; e 50 crianças $(19,7 \%)$ de 11 anos a 11 anos e 11 meses.

\section{Material}

O instrumento utilizado na obtenção da distância interincisiva máxima foi o paquímetro eletrônico digital série 727 da marca Starrett, fabricado no Brasil, feito de aço inoxidável e com mostrador de cristal líquido com indicação do sistema de unidade em milímetros $(\mathrm{mm})$, com resolução e reprodutibilidade de $0,01 \mathrm{~mm}$. Foram utilizados protocolos de coleta de dados, algodão e álcool etílico hidratado.

\section{Procedimento}

$\mathrm{Na}$ primeira etapa, o médico otorrinolaringologista realizou a avaliação otorrinolaringológica, composta por exame físico e radiológico. O exame físico foi constituído de oroscopia para avaliação do tamanho das tonsilas palatinas e rinoscopia anterior para avaliação dos cornetos, septo e mucosa nasal. Foram solicitados exames complementares, como radiografia de cavum, para determinação do grau de obstrução da nasofaringe pela tonsila faríngea. As crianças que, após avaliação otorrinolaringológica e resultados dos exames, receberam o diagnóstico médico de respiração bucal, foram encaminhadas para a avaliação da distância interincisiva máxima. Ao respeitar todos os critérios para participar desse estudo, foi solicitado aos pais ou responsáveis das crianças o preenchimento do termo de consentimento pós-informado. A pesquisa e o termo de consentimento foram aprovados pela Comissão de Ética para Análise de Projetos de Pesquisa (CAPPesq) da Diretoria Clínica do Hospital das Clínicas e da Faculdade de Medicina da Universidade de São Paulo (protocolo de pesquisa n ${ }^{\circ}$ 096/04).

$\mathrm{Na}$ segunda etapa, foi efetuada a coleta da distância interincisiva máxima, que correspondeu à distância entre a borda incisal do dente incisivo central superior direito e a borda incisal do dente incisivo central inferior direito, após a abertura máxima da boca. Quando observou-se dentes incisivos centrais superiores e/ou inferiores direitos em processo de irrupção ou ausentes, foi medida a distância entre os dentes correspondentes do lado esquerdo. Ao se verificar dentes incisivos centrais superiores e/ou inferiores, direitos e esquerdos, em processo de irrupção ou ausentes, foi medida a distância entre a borda incisal do dente incisivo lateral superior direito e a borda incisal do dente incisivo lateral inferior direito. $\mathrm{Na}$ impossibilidade de se obter essa última medida, em decorrência dos dentes incisivos centrais e laterais do lado direito estarem em processo de irrupção ou ausentes, foi medida a distância entre a borda incisal do dente incisivo lateral superior esquerdo e a borda incisal do dente incisivo lateral inferior esquerdo. $\mathrm{Na}$ ausência dos incisivos centrais e laterais inferiores e superiores, ou os mesmos estarem em processo de irrupção, essa medida não foi obtida. 
Foi solicitado à criança que permanecesse sentada, com os pés apoiados no chão, com a cabeça em posição natural. A distância interincisiva máxima foi coletada em vista frontal, com as hastes do paquímetro para medição interna, e transcrita em milímetros para o protocolo de coleta de dados. A medida foi realizada duas vezes e, posteriormente, foi calculada a média aritmética das duas medidas. Ao término da avaliação de cada criança, as hastes do paquímetro foram lavadas e desinfetadas com álcool etílico hidratado, friccionando com algodão.

Foram realizadas, ao final, as análises que compararam os achados referentes à distância interincisiva máxima entre as crianças respiradoras bucais e as crianças sem queixas fonoaudiológicas, verificando se havia diferença estatisticamente significativa entre as médias dessas duas populações, segundo a idade. Os procedimentos de coleta de dados foram semelhantes com as crianças sem queixas fonoaudiológicas ${ }^{5,6,7}$.

\section{Análise estatística}

A população de estudo foi caracterizada por meio de estatística descritiva (média, desvio-padrão, mediana e valores mínimo e máximo). Foi avaliada a aderência à curva Normal pelo uso do teste Komolgorov-Smirnov.

$\mathrm{Na}$ análise dos dados relativos à distância interincisiva máxima, foi realizada a comparação das médias, segundo a idade, por meio da análise de variância (ANOVA) a um fator. Foi calculado o intervalo de $95 \%$ de confiança, segundo a idade.

Foi realizada a comparação das médias da distância interincisiva máxima entre as crianças respiradoras bucais e as crianças sem queixas fonoaudiológicas, segundo a idade, por meio do teste t-Student.

Todas as análises foram realizadas utilizando o pacote estatístico SPSS 12.0 (Statistical Package for the Social Sciences, Chicago, EUA), sendo considerado o nível de significância de $5 \%$.

\section{RESULTADOS}

A estatística descritiva para a distância interin- cisiva máxima, considerando o número total de crianças respiradoras bucais, aponta que a média foi de $43,55 \mathrm{~mm}$, sendo a mediana de $43,75 \mathrm{~mm}$. O valor mínimo obtido foi $31,12 \mathrm{~mm}$ e o valor máximo foi $55,83 \mathrm{~mm}$. Observa-se que a média da distância interincisiva máxima na amostra apresentou valor próximo da mediana e teve aderência à distribuição Normal ( $\mathrm{p}>0,663$ pelo teste de Kolmogorov-Smirnov).

A estatística descritiva para a distância interincisiva máxima, considerando o número total de crianças sem queixas fonoaudiológicas, aponta que a média da distância interincisiva máxima foi $44,75 \mathrm{~mm}$, sendo que a mediana foi $44,65 \mathrm{~mm}$. O valor mínimo obtido foi $32,34 \mathrm{~mm}$, sendo que o valor máximo foi $58,01 \mathrm{~mm}$.

No que se refere à caracterização da população de estudo de acordo com o diagnóstico otorrinolaringológico principal, segundo a idade, tem-se que o mais frequente foi o aumento de tonsila faríngea e de tonsilas palatinas (48\%), seguido do aumento de tonsila faríngea (32\%). O aumento de tonsilas palatinas, bem como a rinite, esteve presente em $15 \%$ da população de estudo. Não foi encontrada diferença estatisticamente significativa entre as porcentagens do diagnóstico otorrinolaringológico, de acordo com a idade ( $p>0,05)$.

Na tabela 1, pode-se observar que não houve diferença estatisticamente significativa entre as médias da distância interincisiva máxima, segundo a idade $(p=0,950)$. O valor mínimo encontrado na amostra foi de $31,12 \mathrm{~mm}$, em uma criança com 7 anos de idade, e o valor máximo foi $55,83 \mathrm{~mm}$, em uma criança com 10 anos de idade.

Na tabela 2, está apresentada a comparação das médias relativas à distância interincisiva máxima. Não foi verificada diferença estatisticamente significativa entre as médias dessa medida nas duas populações estudadas. Os valores médios obtidos nas crianças sem queixas fonoaudiológicas encontram-se próximos aos obtidos para as crianças respiradoras bucais, em todas as faixas etárias ( $\mathrm{p}>0,05)$. 
TABELA 1 - Estatística descritiva da distância interincisiva máxima, segundo a idade.

\begin{tabular}{ccccc}
$\begin{array}{c}\text { IDADE } \\
\text { (anos) }\end{array}$ & $\begin{array}{c}\text { MÉDIA } \\
\text { (mm) }\end{array}$ & $\begin{array}{c}\text { ERRO } \\
\text { PADRÃO }\end{array}$ & $\begin{array}{c}\text { IC 95\% } \\
\text { (média) }\end{array}$ & $\begin{array}{c}\text { MíN. MÁX. } \\
\text { (mm) }\end{array}$ \\
\hline 7 & 43,29 & 0,92 & $41,39-45,19$ & $31,12-51,05$ \\
\hline 8 & 43,85 & 1,07 & $41,61-46,09$ & $31,55-51,67$ \\
\hline 9 & 42,99 & 0,98 & $40,94-45,04$ & $35,24-52,20$ \\
\hline 10 & 43,86 & 1,77 & $39,94-47,77$ & $36,07-55,83$ \\
\hline 11 & 44,20 & 1,18 & $41,63-46,77$ & $35,40-53,04$ \\
\hline
\end{tabular}

IC = intervalo de confiança (ANOVA) $p=0,950$.
TABELA 2 - Comparação entre as médias da distância interincisiva máxima de diferentes populações, segundo a idade.

\begin{tabular}{cccccc} 
IDADE & $n$ & $\begin{array}{c}\text { CRIANÇAS SEM } \\
\text { QUEIXAS }\end{array}$ & n $\begin{array}{r}\text { CRIANÇAS } \\
\text { RESPIRAD0 } \\
\text { RAS BUCAIS }\end{array}$ & p \\
média (mm) & & média (mm) & \\
\hline 7 & 48 & 43,90 & 31 & 43,29 & $\mathrm{p}>0,05$ \\
8 & 51 & 43,70 & 21 & 43,85 & $\mathrm{p}>0,05$ \\
9 & 50 & 45,67 & 21 & 42,99 & $\mathrm{p}>0,05$ \\
10 & 54 & 44,96 & 12 & 43,86 & $\mathrm{p}>0,05$ \\
11 & 50 & 45,46 & 14 & 44,20 & $\mathrm{p}>0,05$
\end{tabular}

$\mathrm{n}=$ número de pacientes; $\mathrm{p}=$ teste $\mathrm{t}$-Student.

A dentadura mista foi o período escolhido, pois algumas investigações ${ }^{5,6,7}$ apontam essa fase da dentição como critério de inclusão, com a presença dos quatro primeiros molares permanentes irrompidos, uma vez que importantes transformações ocorrem na cavidade bucal entre os 5 e 6 anos de idade, em decorrência da irrupção desses dentes. Assim, foi possivel obter uma amostra homogênea quanto ao desenvolvimento da dentição.

Entre os respiradores bucais, não foi verificada diferença estatisticamente significativa entre as médias da distância interincisiva máxima segundo a idade, sendo que a média na amostra foi de $43,55 \mathrm{~mm}$. Notou-se que os resultados obtidos estão de acordo com os dados descritos por Bianchini $^{4}$, que apontam que a abertura máxima da boca inferior a $35 \mathrm{~mm}$, na criança, é um indicativo de possíveis alterações musculares e/ou articulares. Vale ressaltar, entretanto, que existe diferença entre a medida da distância interincisiva máxima e da abertura máxima da boca, dados os diferentes procedimentos para obtê-las.

Os achados referentes à distância interincisiva máxima para as crianças respiradoras bucais estão próximos dos dados publicados para crianças sem queixas fonoaudiológicas $(43,55 \mathrm{~mm}$ e $44,75 \mathrm{~mm}$, respectivamente $)^{6}$. Tais pesquisas respeitaram os mesmos procedimentos de avaliação, possibilitando a comparação dos resultados. 
Ao comparar a distância interincisiva máxima das duas populações estudadas, observa-se que não foi verificada diferença estatisticamente significativa entre as médias, segundo a idade, indicando que nos respiradores bucais essa medida parece não estar comprometida.

Vale ressaltar, por fim, que os respiradores bucais que participaram desse estudo são oriundos de um hospital terciário, sendo que a generalização dos dados obtidos nessa pesquisa pode apresentar-se limitada. Nesse sentido, seria interessante não só duplicar esse tipo de estudo em outros serviços de atenção à saúde, mas também com diferentes populações e em outros períodos da dentição.

No que se refere às considerações finais, temse que o uso do paquímetro mostra-se útil na avaliação fonoaudiológica, complementando o julgamento visual com medidas quantitativas.
É relevante apontar que o seu uso necessita de padronização quanto aos procedimentos, para que se obtenha uma análise precisa da distância interincisiva máxima.

\section{CONCLUSÕES}

1) Não houve diferença estatisticamente significativa entre as médias para a distância interincisiva máxima das crianças respiradoras bucais, segundo a idade.

2) Não houve diferença estatisticamente significativa entre as médias da distância interincisiva máxima dos respiradores bucais e as médias dessa medida de crianças sem queixas fonoaudiológicas, segundo a idade.

\title{
Maximum interincisal distance in mouth breathing children
}

\begin{abstract}
Introduction: The maximum interincisal distance is an important aspect in the orofacial myofunctional evaluation, because orofacial myofunctional disorders can limit the mouth opening. Aim: To describe the maximum interincisal distance of the mouth breathing children, according to age, and to compare the averages of the maximum interincisal distance of mouth breathing children to those of children with no history of speech-language pathology disorders. Methods: Ninety-nine mouth breathing children participated, of both genders, with ages ranging from 7 to 11 years and 11 months, Caucasian, in mixed dentition. The control group was composed by 253 children, with ages ranging from 7 years to 11 years and 11 months, Caucasian, in mixed dentition period, with no history of speech-language pathology disorders. Results: The results show that the average of the maximum interincisal distance of the mouth breathing children was, considering the total sample, 43.55 millimeters, and it did not show statistically significant difference between averages according to age. There is no statistically significant difference between the maximum interincisal distance's averages of the mouth breathing children and the averages of this distance of the control group children. Conclusions: The maximum interincisal distance is one measure that did not modify in mouth breathing children, during mixed dentition period, according to age, and seems not to be altered in this population. It is also observed the importance of use of the caliper in objective evaluation of the maximum interincisal distance.
\end{abstract}

Keywords: Face. Measurements. Mouth. Child. Mouth breathing. 


\section{REFERÊNCIAS}

1. ALMEIDA, P. D. Tratamento ortodôntico: a relação entre respiração oral e crescimento facial. In: KRAKAUER, L. H.; D FRANCESCO, R. C.; MARCHESAN, I. Q. (Org.). Respiração oral. São José dos Campos: Pulso, 2003. p. 151-161.

2. BERRETIN-FELIX, G.; JORGE, T. M.; GENARO, K. F. Intervenção fonoaudiológica em pacientes submetidos à cirurgia ortognática. In: FERREIRA, L. P.; BEFI-LOPES, D. M.; LIMONGI, S. C. O. (Org.). Tratado de Fonoaudiologia. São Paulo: Roca, 2004. p. 494-511.

3. BIANCHINI, E. M. G. Avaliação fonoaudiológica da motricidade oral: anamnese, exame clínico, o quê e por que avaliar. In: _. (Org.). Articulação temporomandibular: implicações, limitações e possibilidades fonoaudiológicas. Carapicuíba: Pró-Fono, 2000. p. 191-253.

4. BIANCHINI, E. M. G. Mastigação e ATM: avaliação e terapia. In: MARCHESAN, I. Q. Fundamentos em Fonoaudiologia: aspectos clínicos da motricidade oral. Rio de Janeiro: Guanabara Koogan, 1998. p. 37-49.

5. CATTONI, D. M. Exame fonoaudiológico: medidas faciais em crianças leucodermas sem queixas fonoaudiológicas. 2003. 213 f. Dissertação (Mestrado em Ciências)-Universidade de São Paulo, São Paulo, 2003.

6. CATTONI, D. M.; FERNANDES, F. D. M. Distância interincisiva máxima em crianças na dentadura mista. R. Dental Press Ortodon. Ortop. Facial, Maringá, v. 10, n. 1, p. 117-121, 2005.

7. CATTONI, D. M.; FERNANDES, F. D. M. Medidas e proporções faciais em crianças: contribuições para a avaliação miofuncional orofacial. Pró-Fono, São Paulo, v. 16, n. 1, p. 7-18, 2004.

8. CHIAPPETTA, A. L. M. L.; ODA, A. L. Doenças neuromusculares. In: FERREIRA, L. P.; BEFI-LOPES, D. M.; LIMONGI, S. C. O. (Org.). Tratado de Fonoaudiologia. São Paulo: Roca, 2004. p. 330-342.

9. COSTA, T. L. S.; SILVA, H. J.; CUNHA, D. A. Análise qualitativa inter-observadores e avaliação morfométrica do palato duro. Rev. Cefac., São Paulo, v. 7, n. 3, p. 326-335, 2005.

10. DI FRANCESCO, R. C. Conseqüências da respiração oral. In: KRAKAUER, L. H.; DI FRANCESCO, R. C.; MARCHESAN, I. Q (Org.). Respiração oral. São José dos Campos: Pulso, 2003. p. 19-25.

11. FARKAS, L. G.; KATIC, M. J.; FORREST, C. R.; LITSAS, L. Surface anatomy of the face in Down's syndrome: Linear and angular measurements in the craniofacial regions. J. Craniofac. Surg., Boston, v. 12, no. 4, p. 373-379, 2001.

12. FERRARIO, V. F.; DELLAVIA, C.; ZANOTTI, G.; SFORZA, C. Soft tissue facial anthropometry in Down syndrome subjects. J. Craniofac. Surg., Boston, v. 15, no. 3, p. 528-532, 2004.

13. FRAZÃO, Y. S. Disfagia na paralisia cerebral. In: FERREIRA, L. P.; BEFI-LOPES, D. M.; LIMONGI, S. C. O. (Org.). Tratado de Fonoaudiologia. São Paulo: Roca, 2004. p. 370-385.

14. GENARO, K. F.; YAMASHITA, R. P.; TRINDADE, I. E. K. Avaliação clínica e instrumental na fissura labiopalatina. In: FERREIRA, L. P.; BEFI-LOPES, D. M.; LIMONGI, S. C. O. (Org.). Tratado de Fonoaudiologia. São Paulo: Roca, 2004. p. 456-477.

15. GUEDES, Z. C. R. Alterações oromiofuncionais em síndromes. In: FERREIRA, L. P.; BEFI-LOPES, D. M.; LIMONGI, S. C. O (Org.). Tratado de Fonoaudiologia. São Paulo: Roca, 2004. p. $254-260$.
16. HAMAZAKI, C. M. et al. Verificação da amplitude dos movimentos mandibulares em crianças. Rev. Cefac., São Paulo, v. 4, n. 1, p. 35-39, 2002.

17. JARDINI, R. S. R. Uso do exercitador labial: estudo preliminar para alongar e tonificar os músculos orbiculares orais. Pró-Fono, São Paulo, v. 11, n. 1, p. 8-12, 1999.

18. JUNQUEIRA, P. Avaliação e diagnóstico fonoaudiológico em motricidade oral. In: FERREIRA, L. P.; BEFI-LOPES, D. M.; LIMONGI, S. C. O. (Org.). Tratado de Fonoaudiologia. São Paulo: Roca, 2004. p. 230-236.

19. JUNQUEIRA, P. Avaliação miofuncional. In: MARCHESAN, I. Q. Fundamentos em Fonoaudiologia: aspectos clínicos da motricidade oral. Rio de Janeiro: Guanabara Koogan, 1998. p. 13-21.

20. MARCHESAN, I. Q. Avaliação e terapia dos problemas da respiração. In: ___. Fundamentos em Fonoaudiologia: aspectos clínicos da motricidade oral. Rio de Janeiro: Guanabara Koogan, 1998. p. 23-36.

21. MARCHESAN, I. Q. Protocolo de avaliação miofuncional orofacial. In: KRAKAUER, L. H.; DI FRANCESCO, R. C.; MARCHESAN, I. Q. (Org.). Respiração oral. São José dos Campos: Pulso, 2003. p. 55-79.

22. MARCHESAN, I. Q. The speech pathology treatment with alterations of the stomatognathic system. Int. J. Orofacial Myology, Seattle, v. 26, no. 2, p. 5-12, 2000

23. RÍSPOLI, C. M.; BACHA, S. M. C. Terapia miofuncional: intervenção fonoaudiológica breve. In: MARCHESAN, I. Q.; ZORZI, J. L.; GOMES, I. C. (Org.). Tópicos em Fonoaudiologia. São Paulo: Lovise, 1998. p. 545-585.

24. RODRIGUES, K. A.; LEFĖVRE, A. P.; MOTT, L. B.; TUGUMIA, D.; PENA, P. L. Análise comparativa entre o lado de predominância mastigatória e medidas da mandíbula por meio do paquímetro. Rev. Cefac., São Paulo, v. 5, n. 4, p. 347-351, 2003.

25. RODRIGUES, L. Avaliação odontológica. In: BIANCHINI, E. M. G. (Org.). Articulação temporomandibular: implicações, limitações e possibilidades fonoaudiológicas. Carapicuíba: PróFono, 2000. p. 133-166.

26. SEIKEL, J. A.; KING, D. W.; DRUMRIGHT, D. G. Physiology of mastication and deglutition. In: SEIKEL, J. A.; KING, D. W.; DRUMRIGHT, D. G. Anatomy \& physiology for speech, language, and hearing. 3rd ed. New York: Thomson, 2005. p. 391-405.

27. SILVA, H. J.; CUNHA, D. A. Considerações sobre o uso do paquímetro em motricidade oral. Fonoaudiol. Brasil, Brasília, DF, v. 2, n. 4, p. 59-64, 2003.

28. SOUZA, L. C. M.; CAMPIOTTO, A. R.; FREITAS, R. R. Cirurgia Ortognática e Fonoaudiologia. In: LOPES FILHO, O. (Ed.). Tratado de Fonoaudiologia. São Paulo: Roca, 1997. p. 781-804.

29. TESSITORE, A. Alterações oromiofuncionais em respiradores orais. In: FERREIRA, L. P.; BEFI-LOPES, D. M.; LIMONGI, S. C. O. (Org.). Tratado de Fonoaudiologia. São Paulo: Roca, 2004. p. 261-276.

30. WARD, R. E.; JAMISON, P. L.; ALLANSON, J. E. Quantitative approach to identifying abnormal variation in human face exemplified by a study of 278 individuals with five craniofacial syndromes. Am. J. Med. Genet., New York, v. 91, no. 1, p. 8-17, 2000.
Endereço para correspondência

Débora Martins Cattoni

Rua Barão da Passagem, 1330 apto. 91C

CEP: 05.087-000 - São Paulo / SP

E-mail: dmcattoni@uol.com.br 